\title{
Mass composition of the escaping plasma at Mars
}

August 25, 2005

E. Carlsson ${ }^{a, b}$, A. Fedorov ${ }^{c}$, S. Barabash ${ }^{a}$, E. Budnik ${ }^{c}$, A. Grigoriev ${ }^{a}$, H. Gunell ${ }^{a}$, H. Nilsson ${ }^{a}$, J.-

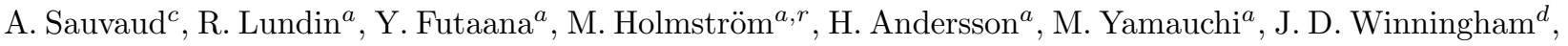
R. A. Frahm ${ }^{d}$, J. R. Sharber ${ }^{d}$, J. Scherrer ${ }^{d}$, A. J. Coates $^{e}$, D. R. Linder ${ }^{e}$, D. O. Kataria ${ }^{e}$, E. Kallio $^{f}$, H. Koskinen $^{f}$, T. Säles ${ }^{f}$, P. Riihela ${ }^{f}$, W. Schmidt ${ }^{f}$, J. Kozyra ${ }^{g}$, J. Luhmann $^{h}$, E. Roelof ${ }^{i}$, D. Williams ${ }^{i}$, S. Livi $^{i}$, C. C. Curtis $^{j}$, K. C. Hsieh ${ }^{j}$, B. R. Sandel ${ }^{j}$, M. Grande ${ }^{k}$, M. Carter ${ }^{k}$, J.-J. Thocaven ${ }^{c}$, S. McKenna-Lawlor ${ }^{l}$,

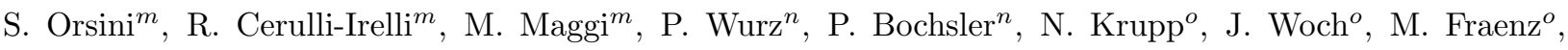
K. Asamura ${ }^{p}$, C. Dierker ${ }^{q}$

${ }^{a}$ Swedish Institute of Space Physics, Box 812, SE-981 28 Kiruna, Sweden

${ }^{b}$ Luleå University of Technology, Luleå, Sweden

${ }^{c}$ Centre d'Etude Spatiale des Rayonnements, BP-4346, F-31028 Toulouse, France

${ }^{d}$ Southwest Research Institute, San Antonio, TX 7228-0510, USA

${ }^{e}$ Mullard Space Science Laboratory, University College London, Surrey RH5 6NT, UK

${ }^{f}$ Finnish Meteorological Institute, Box 503 FIN-00101 Helsinki, Finland

${ }^{g}$ Space Physics Research Laboratory, University of Michigan, Ann Arbor, MI 48109-2143, USA

${ }^{h}$ Space Science Laboratory, University of California at Berkeley, Berkeley, CA 94720-7450, USA

${ }^{i}$ Applied Physics Laboratory, Johns Hopkins University, Laurel, MD 20723-6099, USA

${ }^{j}$ University of Arizona, Tucson, AZ 85721, USA

${ }^{k}$ Rutherford Appleton Laboratory, Chilton, Didcot, Oxfordshire OX11 0QX, UK

${ }^{l}$ Space Technology Ireland, National University of Ireland, Maynooth, Co. Kildare, Ireland.

${ }^{m}$ Instituto di Fisica dello Spazio Interplanetari, I-00133 Rome, Italy

${ }^{n}$ University of Bern, Physikalisches Institut, CH-3012 Bern, Switzerland

${ }^{\circ}$ Max-Planck-Institut fur Aeronomie, D-37191 Katlenburg-Lindau, Germany 
${ }^{p}$ Institute of Space and Astronautical Science, 3-1-1 Yoshinodai, Sagamichara, Japan

${ }^{q}$ Technical University of Braunschweig, Hans-Sommer-Strasse 66, D-38106 Braunschweig, Germany

${ }^{r}$ Currently at NASA Goddard Space Flight Center, Laboratory for Solar and Space Physics, Mail Code 612.2, Greenbelt, MD 20771, USA 
Corresponding author: Ella Carlsson

Mailing address: IRF, Box 812, 981 28, Kiruna, SWEDEN

Telephone: + 46 (0) $980-79124$

Email: ella@irf.se

Running title: Mass composition of the escaping plasma at Mars 


\begin{abstract}
Data from the Ion Mass Analyser (IMA) sensor of the ASPERA-3 instrument suite on Mars Express have been analysed to determine the mass composition of the escaping ion species at Mars. We have examined 77 different ion-beam events and we present the results in terms of flux ratios between the following ion species: $\mathrm{CO}_{2}^{+} / \mathrm{O}^{+}$and $\mathrm{O}_{2}^{+} / \mathrm{O}^{+}$. The following ratios averaged over all events and energies were identified: $\mathrm{CO}_{2}^{+} / \mathrm{O}^{+}=0.2$ and $\mathrm{O}_{2}^{+} / \mathrm{O}^{+}=0.9$. The values measured are significantly higher, by a factor of 10 for $\mathrm{O}_{2}^{+} / \mathrm{O}^{+}$, than a contemporary modeled ratio for the maximum fluxes which the Martian ionosphere can supply. The most abundant ion species was found to be $\mathrm{O}^{+}$, followed by $\mathrm{O}_{2}^{+}$and $\mathrm{CO}_{2}^{+}$. We estimate the loss of $\mathrm{CO}_{2}^{+}$to be $4.0 \cdot 10^{24} \mathrm{~s}^{-1}\left(0.29 \mathrm{~kg} \mathrm{~s}^{-1}\right)$ by using the previous measurements of Phobos-2 in our calculations. The dependence of the ion ratios in relation to their energy ranges we studied, 0.3-3.0 keV, indicated that no clear correlation was found.
\end{abstract}

Keywords: Martian ionosphere, flux ratios, escape 


\section{Introduction}

Geomorphological evidence in form of riverbeds [Malin and Edgett, 2003], gullies [Malin and Edgett, 2000] and shorelines [Head et al., 1999], as well as recently discovered hematite spheres [Calvin, 2004] and a frozen sea [Murray, 2005], suggest that there has been liquid water in the past on the Martian surface. In order for these features to form, a dense $\mathrm{CO}_{2}$ atmosphere of a few bars including gases of $\mathrm{CH}_{4}$ and $\mathrm{NH}_{3}$ [Kasting, 1991] would be required to produce the necessary greenhouse effect. The present $\mathrm{CO}_{2}$ atmosphere pressure is only 7-9 mbars [Hess et al., 1979]. Moreover, spectral imaging of Mars clearly indicates that the amount of carbonates stored at Mars in the form of ice and carbonate rocks is too insignificant to explain the relatively dense atmosphere that existed in the past [Bibring et al., 2005]. This discrepancy led us to investigate the mass composition of the escaping plasma at Mars in order to determine if there might be an abundance of escaping ion species incorporating carbon, such as $\mathrm{CO}_{2}^{+}$.

Up to an altitude of $150 \mathrm{~km}, \mathrm{CO}_{2}$ is the dominant constituent in the atmosphere of Mars, supplying $\mathrm{CO}_{2}^{+}$ through photoionization:

$$
\mathrm{CO}_{2}+h \nu \rightarrow \mathrm{CO}_{2}^{+}+e^{-}
$$

However, the main ambient ion in the ionosphere is $\mathrm{O}_{2}^{+}$which also has a peak at $\sim 130 \mathrm{~km}$ with a density of $\sim 10^{5} \mathrm{~cm}^{-3} . \mathrm{O}_{2}^{+}$is formed by several different processes as shown below:

$$
\begin{aligned}
& \text { atom-ion interchange: } \mathrm{O}+\mathrm{CO}_{2}^{+} \rightarrow \mathrm{O}_{2}^{+}+\mathrm{CO} \\
& \text { or charge transfer: } \mathrm{O}+\mathrm{CO}_{2}^{+} \rightarrow \mathrm{O}^{+}+\mathrm{CO}_{2} \\
& \text { rapidly followed by: } \mathrm{O}^{+}+\mathrm{CO}_{2} \rightarrow \mathrm{O}_{2}^{+}+\mathrm{CO}
\end{aligned}
$$

In the martian ionosphere both $\mathrm{CO}_{2}^{+}$and $\mathrm{O}_{2}^{+}$disappear through dissociative recombination:

$$
\begin{gathered}
\mathrm{CO}_{2}^{+}+e^{-} \rightarrow \mathrm{CO}+\mathrm{O} \\
\mathrm{O}_{2}^{+}+e^{-} \rightarrow \mathrm{O}+\mathrm{O}
\end{gathered}
$$


$\mathrm{CO}^{+}$and $\mathrm{CO}_{2}^{+}$exhibit peak densities at $\sim 200 \mathrm{~km}$ and $\sim 140 \mathrm{~km}$, of $\sim 100 \mathrm{~cm}^{-3}$ and $\sim 2 \cdot 10^{4} \mathrm{~cm}^{-3}$, respectively [Fox, 2004].

The maximum upward fluxes of the main ion species as estimated by Fox[2005](private communication) are reproduced in Table 1. It is assumed in this model that all ions have a velocity of $1.2 \cdot 10^{5} \mathrm{~cm} \mathrm{~s}^{-1}$ at the upper model boundary at an altitude of $400 \mathrm{~km}$. In Table 1 it is clearly seen that $\mathrm{O}_{2}^{+}$has the highest predicted flux, followed by $\mathrm{CO}_{2}^{+}$, while $\mathrm{O}^{+}$has the lowest predicted flux rate. This suggests that the most abundant ion species escaping the Martian ionosphere is $\mathrm{O}_{2}^{+}$followed by $\mathrm{CO}_{2}^{+}$and $\mathrm{O}^{+}$.

Ions attaining an altitude above $400 \mathrm{~km}$ are picked up by the solar wind and can escape from Mars through the magnetotail. MHD simulations performed by Liu [2001] indicate that the most abundant ion species in the tailward escaping Martian plasma is $\mathrm{O}_{2}^{+}$, followed by $\mathrm{O}^{+}$with escape rates of $2.61 \cdot 10^{25} \mathrm{~s}^{-1}$ and $0.45 \cdot 10^{25} \mathrm{~s}^{-1}$, respectively giving the flux ratio of $\mathrm{O}_{2}^{+} / \mathrm{O}^{+}$to be 5.8 , which differ $61 \%$ to the fluxes given by Fox [2005](private communication). Ions can escape by means of thermal and most of the non-thermal escape mechanisms such as charge exchange, dissociative recombination and sputtering. The thermal escape of ions normally applies to lighter ions.

In-situ measurements made onboard the satellite Phobos-2 by the instrument ASPERA indicate that the non-thermal escape involves a mixture of ions such as $\mathrm{O}^{+}, \mathrm{O}_{2}^{+}$and $\mathrm{CO}_{2}^{+}$, according to Norberg et al.[1993]. The total ion escape of $\mathrm{O}^{+}$was estimated by Lundin et al.[1989] to be $\sim 2 \cdot 10^{25} \mathrm{~s}^{-1}$, corresponding to a loss rate of $0.5 \mathrm{~kg} \mathrm{~s}^{-1}$. The instrument also detected planetary $\mathrm{H}^{+}$and molecular ions such as $\mathrm{O}_{2}^{+}, \mathrm{CO}_{2}^{+}$ and $\mathrm{CO}^{+}$, which could not be separately resolved due to the insufficient mass resolution of the instrument. Overall the total ion escape was estimated to reach at least $\sim 1 \mathrm{~kg} \mathrm{~s}^{-1}$ according to Lundin et al.[1989].

The study in the present paper is a first attempt to analyse data recorded by the Ion Mass Analyzer (IMA) onboard the satellite Mars Express, in order to determine the relative outflow of the main ionospheric species, with regard in particular to the ions that include carbon. In the Section 2 we explain the measurement technique of the IMA sensor and the following section is devoted to data analysis and methodology. The results of this study are presented in Section 4 and discussed in Section 5. 


\section{The IMA sensor}

The Ion Mass Analyzer (IMA) [Barabash et al., 2004] is almost an exact copy of the Rosetta's ICA instrument and an upgraded version of the ion mass spectrographs TICS/Freja, IMIS/Mars-96 and IMI/Nozomi [Norberg et al., 1998]. The IMA sensor measures the differential ion flow in the energy range 0.01-30 keV/q for ion components that include $\mathrm{H}^{+}, \mathrm{He}^{2+}, \mathrm{O}^{+}$and molecular ions within the range of $20<\mathrm{M} / \mathrm{q}<80$. Electrostatic sweeping provides the sensor with a $\pm 45^{\circ}$ polar angle, which gives the instrument an intrinsic field of view (FOV) of $90^{\circ} \times 360^{\circ}$. The FOV is divided into $16\left(5.6^{\circ}\right.$ each $)$ polar angles and $16\left(22.5^{\circ}\right.$ each $)$ azimuth sectors. When ions within a swept energy pass band enter the IMA sensor they are strained through an outer grounded grid. Behind the grid the ions pass a deflection system whose purpose is to deflect ions in the range of $45^{\circ}$ and $135^{\circ}$ with respect to the symmetry axes into the top-hat electrostatic analyzer (ESA). The ESA permits ions in a swept energy pass band, with an energy resolution of $8 \%$, to enter the mass selection and detection unit. Permanent magnets then deflect the ions along different trajectories, depending on their energy, mass and charge. Lighter ions are deflected further outward from the center than the heavy ions. All of the ions then hit the micro-channel plate (MCP) which has a position sensitive anode composed of the 16 sectors x 32 rings, which determines both the azimuth (sector) and mass per charge of the incoming ion species (mass rings). The magnet assembly can be biased with respect to the ESA, to post-accelerate ions, thereby permitting the selection of mass range and mass resolution. In a mode without any post-acceleration, the sensor has the highest mass resolution but lighter particles with low energies, such as $\mathrm{H}^{+}$, are diverged along their flight paths to such an extent that they miss the MCP altogether and cannot be detected. Post-acceleration up to $4 \mathrm{keV}$ allows the detection of protons (for solar wind observations). However, this broadens the mass-band and limits the mass resolution. The sampling time of the instrument is $125 \mathrm{~ms}$ and a full $3 \mathrm{D}$ spectrum accumulation sweep of 16 polar angles $\times 16$ azimuthal sectors $\times 32$ mass rings $\times 96$ energies requires $192 \mathrm{~s}$ to complete.

\section{Methodology}

\subsection{Identification of planetary ion events}

Strong fluxes of protons in the magnetosheath outside the induced magnetosphere boundary (IMB) may affect mass channels even with M/q > 4 at the same energy. This is because internally scattered particles from the magnets and magnetic section appear as ghosts in the data read-out, thereby complicating the analysis. 
To identify clear events of planetary ions we first examined all orbits traversed during time intervals when Mars Express was eclipsed by Mars, starting on 23 April 2004. By 15 August 2004 the orbital parameters of the satellite were changed to such an extent that the eclipse season ended. However, upon examination of the selected data we came to the conclusion that more measurements were needed in order to achieve better statistics. We then decided to investigate a larger area which included the induced magnetosphere but was still inside the induced magnetosphere boundary to avoid proton contamination from the heavy mass channels in the IMA sensor.

Figure 1 shows the area inside the induced magnetosphere boundary (IMB), on which we concentrated our search of planetary ion events. The orbit of Mars Express is here displayed in cylindrical coordinates expressed in Mars radii, $\mathrm{R}_{m}$. The $x$-axis in this coordinate system is directed toward the sun with the center of Mars as its origin and the orientation of the $y$-axis is according to the right-hand system. The $z$-axis is directed northward, perpendicular to the ecliptic plane. The distance from the satellite to the Mars-Sun line is defined by $\sqrt{y^{2}+z^{2}}$, and indicated as $\mathrm{R}$ in the figure. The solid blue lines in the figure indicate the bow shock (BS) and the induced magnetosphere boundary (IMB). The shape of the bow shock and of the induced magnetosphere boundary were calculated after Kallio[1996] and Lundin et al.[1990], respectively. The shaded blue area indicates the eclipse. Orbit \#539, which occurred on 22 June 2004, is indicated by a red line and the blue dots along this line represent time intervals of ten minutes. The overall time interval 15:30-16:40 in universal time (UT), indicates the area to which we conducted our research to.

Figure 2 shows an example of an event (in orbit \#539), which was selected for further analysis. The figure displays three panels illustrating IMA-data with different ion masses. The lowest panel shows ion masses per charge in the range 0.0-1.5 M/q (protons), the next panel shows 1.5-3 M/q $\left(\mathrm{He}^{2+}\right)$ and the top one shows $12-90 \mathrm{M} / \mathrm{q}$ (heavy ions, $\mathrm{O}^{+}, \mathrm{CO}^{+}, \mathrm{O}_{2}^{+}, \mathrm{CO}_{2}^{+}$). The two lower panels are used for plasma domain identification and the upper one for displaying heavy ion events. In figure 2 two black arrows point to a heavy ion event, typical of those analysed in the present study, which was identified in the time interval 16:03-16:08 UT. The vertical axis for each panel in the figure shows the energy per charge in $\mathrm{eV}$ and in this particular event the beam of heavy ion species had an energy of $800 \mathrm{eV}$. The colour indicates the count rate, where blue represents the lowest count rate measured and red represents the highest. Cases where the count rates were higher than 100, are represented in black, as seen in figure 2. The full 3D spectrum sweep lasted for $192 \mathrm{~s}$ and the effects of it can be seen in the figure. This modulation appears as an increase in 
counts of ion species in clusters for periods of $192 \mathrm{~s}$. The internal scattering of protons may induce cross talk in the instrument, which can be seen in the upper panel in figure 2, where counts from the protons fluxes appear as ghosts in high energy ranges. The two vertical lines in the figure indicate crossings of the induced magnetosphere boundary. The inbound crossing occurs at 15:38 UT and the outbound crossing at 16:44 UT. The inbound crossing of the induced magnetosphere boundary is very well distinguished by a decrease in proton fluxes. Similarly, the outbound crossing is especially easy to recognise due to its association with a well defined increase in proton fluxes, which has been observed for all orbits. The two dashed lines indicate the beginning and the end of the geometrical eclipse. The start of the eclipse is marked by a momentary decrease in the detected particle fluxes. Beams of heavy ions can clearly be seen inside the eclipse area in figure 2, which we consider to be a valid ion-beam event.

In addition to inspecting data from the IMA sensor, we also examined data from the electron spectrometer (ELS), which is part of the ASPERA-3 instrument. ELS measures electron fluxes in the energy range 0.01-20 keV. These data indicate higher count rates of electrons when there is an increase in ion counts, especially when we identified an ion-beam event. The higher count rate in electrons also served as an indication showing that the ion-beam event could not be an instrument affect.

Figure 3 shows a typical energy-mass matrix of ion-beam events in this study. The event of orbit \#539 is here integrated over the event time interval, selected energy steps, polar and azimuthal directions, which are showing the highest count rate. The vertical axis represents the energy per charge in $\mathrm{eV}$ and the horizontal axis represents the IMA sensor's mass ring number. The red and blue lines in the figure indicate the calibrated mass band ranges for different ion species of $\mathrm{O}_{2}^{+}, \mathrm{O}^{+}, \mathrm{He}^{2+}$ and $\mathrm{H}^{+}$. The lower count rate from mass ring number 22 is a result of the anode read-out electronics priority scheme and was disregarded in the analysis. The highest ion count for this particular heavy ion-beam event was found for the polar angles of 5-15 and the azimuth angles of 1-4. Note that the angular distribution of the planetary ion flow is very narrow, usually less than $20^{\circ}$, e.i. one azimuthal sector. The mass peak of this ion-beam event lies at an energy of $\sim 800 \mathrm{eV}$ and occupies both mass bands of $\mathrm{O}_{2}^{+}$and $\mathrm{O}^{+}$. There is no obvious separation between these two species and we need to apply a special technique to reconstruct each mass distribution. 


\subsection{Mass composition analysis}

The IMA sensor is designed and optimised for studies of plasma dynamics. Since it does not meet the same specifications as a mass spectrometer, it has a reduced capacity to directly resolve and separate adjacent ion masses. Figure 3 shows that even $\mathrm{O}_{2}^{+}$and $\mathrm{O}^{+}$are not well separated. The main approach in separating the different mass species is based on the following two facts: 1) For each energy and for each given mass, the central position of the mass peak has been obtained from ground calibrations, and 2) the shape of the peak is always Gaussian with a constant characteristic width which also was obtained from calibrations on ground and later justified in flight data. Thus for each ion-beam event and each energy we try to fit the shape of the mass spectra with three Gaussian fittings, corresponding to $\mathrm{O}^{+}(\mathrm{M} / \mathrm{q}=16), \mathrm{O}_{2}^{+}(\mathrm{M} / \mathrm{q}=32)$, and $\mathrm{CO}_{2}^{+}(\mathrm{M} / \mathrm{q}=44)$. Note that only the height of the peak is the subject of the best fit of the in-flight data. The position and width is known from calibration data. This fitting technique was developed from a program made by Markwardt[2003] to create the best Gaussian mass-fitting for a post-acceleration of $2.4 \mathrm{kV}$. Figure 4 displays an example of such a mass peak reconstruction. The top left panel in figure 4 shows count rates as a function of mass ring for different energies for the ion-beam event identified during orbit \#539. The other three panels display the Gaussian fittings of the recorded count rates that were performed to resolve the different ion masses for each energy. The best fit for this ion-beam event occurred in the energy range of $600-900 \mathrm{eV}$. The identified ion species in this particular event are $\mathrm{O}_{2}^{+}$with a peak count rate of 190 at $733.9 \mathrm{eV}$, followed by $\mathrm{O}^{+}$. Note that the wings of the $\mathrm{O}_{2}^{+}$distribution are composed of both $\mathrm{CO}_{2}^{+}$and $\mathrm{O}^{+}$.

In figure 5, we can see the signature of an ion-beam event that occurred on 10 June 2004 at 07:52-08:10 UT. The most abundant species in this event is $\mathrm{O}^{+}$, with a peak count rate of 70 and with an energy of 1025.7 $\mathrm{eV}$. The minor ion species in this event is $\mathrm{O}_{2}^{+}$, visible as a lag on the $\mathrm{O}^{+}$peak. The area under the $\mathrm{O}^{+}$peak is marked with red lines in the figure. Within this area we counted all the ion counts coming from polar angles 5-15 $\left(61.9^{\circ}\right)$ and azimuth angles 1-4 $\left(90^{\circ}\right)$ over the time interval of 18 minutes. The total count for the specified time interval, energy, polar- and azimuth angles was 430 for $\mathrm{O}^{+}$and $82 \mathrm{for}_{2}^{+}$for this event. We performed this total ion count procedure for all the mass-fitted areas and for every energy in each event.

Figure 6 displays the orbital positions over which we encountered the ion-beam events. To avoid any possible mass channel contaminations from protons we considered events only inside the induced magnetosphere boundary. Most of the detected events occurred between 1-2 Martian radii from the planet on the night side, as seen in the figure. 
We investigated all orbits from 23 April 2004 to 31 December 2004 inside the induced magnetosphere and examined them for heavy ion events. We discarded all ion-beam events with: (1) fewer ion counts than 25, (2) events with no post-acceleration and (3) events characterised by poor Gaussian mass-fitting, which were selected manually. Altogether we found 77 ion-beam events, each with one or more energy levels, adding up to a total of 209 mass-fitted ion-beam events.

\section{Results}

First we calculate all the ratios of the $\mathrm{CO}_{2}^{+} / \mathrm{O}^{+}$and $\mathrm{O}_{2}^{+} / \mathrm{O}^{+}$fluxes for each event following the standard relation between differential flux and count rate for an electrostatic analyzer [Kessel, 1989]:

$$
\begin{aligned}
R_{j}\left(C O_{2}^{+} / O^{+}\right) & =\frac{\sum_{i} \frac{N_{i j}\left(C O_{2}^{+}\right)}{E_{i j}}}{\sum_{i} \frac{N_{i j}\left(O^{+}\right)}{E_{i j}}} \\
R_{j}\left(O_{2}^{+} / O^{+}\right) & =\frac{\sum_{i} \frac{N_{i j}\left(O_{2}^{+}\right)}{E_{i j}}}{\sum_{i} \frac{N_{i j}\left(O^{+}\right)}{E_{i j}}}
\end{aligned}
$$

where the index $i$ denotes the energy step, $j$ the event number, $E_{i j}$ the energy and $N_{i j}$ the integrated counts under the fitted peak for respective ion.

Then we investigate if the ratios of the $\mathrm{CO}_{2}^{+} / \mathrm{O}^{+}$and $\mathrm{O}_{2}^{+} / \mathrm{O}^{+}$fluxes have any correlation with energy. First we calculate the mean energy for each event with regard to the number of counts under the fitted $\mathrm{O}^{+}$ peaks by:

$$
<E>_{j}\left(O^{+}\right)=\frac{\sum_{i} E_{i j} \frac{N_{i j}\left(O^{+}\right)}{E_{i j}^{2}}}{\sum_{i} \frac{N_{i j}\left(O^{+}\right)}{E_{i j}^{2}}}
$$

where the ratio $N_{i j} / E_{i j}^{2}$ is proportional to the distribution function. The result is presented in figure 7 where it can be seen that there is no clear correlation between energy and ratio. In the upper panel where the ratios of $\mathrm{CO}_{2}^{+} / \mathrm{O}^{+}$are displayed, it can also bee seen that some of the ratios are zero which is due to the absence of $\mathrm{CO}_{2}^{+}$in 12 of the events. 
We then investigate the $\mathrm{CO}_{2}^{+} / \mathrm{O}^{+}$and $\mathrm{O}_{2}^{+} / \mathrm{O}^{+}$ratio distributions (eq.7-8) by displaying them in histograms as seen in figures 8 and 9 . We used a bin-size of 0.1 and applied a logarithmic scale to the vertical axis, which represents the number of ratios. If a particular ratio occurs only once, it is discarded from the histogram and further calculations. It is clearly seen that many of the $\mathrm{CO}_{2}^{+} / \mathrm{O}^{+}$ratios have a value of zero, which corresponds to an absence of $\mathrm{CO}_{2}^{+}$. Note that $\mathrm{O}^{+}$is observed in all of the events.

We then calculated the arithmetic mean for all the ratios that we used in the histograms by:

$$
\begin{gathered}
\overline{R_{j}}\left(C O_{2}^{+} / O^{+}\right)=\frac{1}{N_{\text {ratios }}\left(C O_{2}^{+} / O^{+}\right)} \sum_{j} R_{j}\left(C O_{2}^{+} / O^{+}\right) \\
\overline{R_{j}}\left(O_{2}^{+} / O^{+}\right)=\frac{1}{N_{\text {ratios }}\left(O_{2}^{+} / O^{+}\right)} \sum_{j} R_{j}\left(O_{2}^{+} / O^{+}\right)
\end{gathered}
$$

where $N_{\text {ratios }}$ is the number of respective ratios.

We also estimate the standard deviation for these ratios (eq.10-11) by:

$$
\begin{gathered}
\overline{\Delta R_{j}}\left(C O_{2}^{+} / O^{+}\right)=\left(\frac{1}{N_{\text {ratios }}\left(C O_{2}^{+} / O^{+}\right)-1} \sum_{j}\left(R_{j}\left(C O_{2}^{+} / O^{+}\right)-\overline{R_{j}}\left(C O_{2}^{+} / O^{+}\right)\right)^{2}\right)^{1 / 2} \\
\overline{\Delta R_{j}}\left(O_{2}^{+} / O^{+}\right)=\left(\frac{1}{N_{\text {ratios }}\left(O_{2}^{+} / O^{+}\right)-1} \sum_{j}\left(R_{j}\left(O_{2}^{+} / O^{+}\right)-\overline{R_{j}}\left(O_{2}^{+} / O^{+}\right)\right)^{2}\right)^{1 / 2}
\end{gathered}
$$

which is presented in Table 2. We define a confidence interval for $97 \%$ probability as from 0 to $\overline{R_{j}}+\overline{\Delta R_{j}}$ for the $\mathrm{CO}_{2}^{+} / \mathrm{O}^{+}$flux, because it turns out that $\overline{\Delta R_{j}}>\overline{R_{j}}$.

The most surprising discovery in this study is that the ratio of $\mathrm{O}_{2}^{+} / \mathrm{O}^{+}$diverges with a factor of 10 than the ratios deduced from the maximum upward fluxes modeled by Fox [2005](private communication).

\section{Discussion}

Based on in-situ measurements from the instrument ASPERA onboard the satellite Phobos-2, Lundin et al.[1989] concluded that the total escape rate of $\mathrm{O}^{+}$was $2 \cdot 10^{25} \mathrm{~s}^{-1}$. The ASPERA instrument could measure angular distribution, composition and energy of ions within the energy regions of $0.5 \mathrm{eV} / \mathrm{q}-24 \mathrm{keV} / \mathrm{q}$, which is similar to the IMA sensor. The ion analyzer on Phobos- 2 also had a FOV of $360^{\circ}$ divided into 10 sensors which each had an intrinsic FOV of $3^{\circ} \times 36^{\circ}$. Since Phobos- 2 was spinning with a spin-period of 12 minutes, 
a three-dimensional particle coverage of ions could be obtained. The three-dimensional plasma moments such as flow velocity and number density, needed to calculate the ion escape, were deduced from the twodimensional moments by assuming azimuthal symmetry. Similar ion beam events such as analyzed in this study, were also detected by Phobos-2. However, the ion escape was integrated over the whole magenotail region and just for a couple of orbits. Furthermore, Phobos-2 was functional during high solar activity.

By using this number and the ratio of $\mathrm{CO}_{2}^{+} / \mathrm{O}^{+}$presented in Table 2, we can estimate the escape rate for $\mathrm{CO}_{2}^{+}$to be $4.0 \cdot 10^{24} \mathrm{~s}^{-1}\left(0.29 \mathrm{~kg} \mathrm{~s}^{-1}\right)$ with the escape rate data of $\mathrm{O}^{+}$from Phobos-2. We then extrapolate the numbers back in time with disregard to possible solar wind variations due to the faint young sun. By using this very tentative model we find the total loss of $\mathrm{CO}_{2}^{+}$over the past 4 billion years to be $3.7 \cdot 10^{16}$ kg. However, ion escape from the Martian atmosphere is effected by the rate of photoionization and the temperature of the exosphere which strongly depends on the solar XUV radiation which was $\sim 100-1000$ times stronger for the young-sequence sun [Ribas, 2005]. Furthermore, the young solar wind may have been more than 1000 times more massive than today [Wood, 2002]. Both the increased XUV radiation and the higher solar wind density and velocity in the past suggest that the solar wind erosion must have had a dramatic impact on the evolution of the Martian atmosphere where the ion escape rates must have been significantly higher [Lammer, 2003]. At present Mars lacks an intrinsic magnetic field, however, magnetic anomalies have been detected with concentrations in the southern hemisphere which implies that Mars used to have an intrinsic magnetic field in the past [Acuña, 1998]. This would also have a major impact on the escape rates of ions.

Let us compare the escape rate with the total amount of $\mathrm{CO}_{2}$ in the early atmosphere of Mars. As discussed in section 1, the climate on Mars in the past must have been warmer and wetter in order to explain all the water-related features. According to different models [Haberle, 1998], Mars could have had an atmospheric pressure between $\sim 1-5$ bar in order to raise the surface temperatures above $273 \mathrm{~K}$ needed to create these geomorphological features. If Mars had a pressure of $\sim 3$ bars, then the atmosphere would have a mass of $\sim 1.2 \cdot 10^{19} \mathrm{~kg}$, whereas the atmosphere today (6.36 mbars) only has a mass of $\sim 2.5 \cdot 10^{16} \mathrm{~kg}$. If we compare the mass of the atmosphere in the past and in the present with the rough estimated total escape mass of $\mathrm{CO}_{2}^{+}$, we can conclude that the solar wind and the XUV radiation must have been $\sim 1000$ times higher in order to explain the removal of $\mathrm{CO}_{2}$ from the ancient Martian atmosphere. Impact erosion could also have contributed to the atmospheric loss [Melosh and Vickery, 1989]. 
An other additional factor which may increase the total $\mathrm{CO}_{2}^{+}$escape through the solar wind interaction is the escape in the form of cold ionospheric plasma clouds [Penz et al., 2005]. However, the detection of such clouds is beyond the capability of the IMA sensor. The reason for deviations between the ratios defined by the maximum ionospheric supply and our measurements is not clear. The most probable explanation is that the escape is driven by a mass selection processes which does not reflect the actual ionospheric composition.

\section{Acknowledgments}

The ASPERA-3 experiment on the European Space Agency (ESA) Mars Express mission is a joint effort between 15 laboratories in 10 countries, all sponsored by their national agencies. We thank all these agencies as well as the various departments/institutes hosting these efforts. We also wish to acknowledge the Swedish National Space Board for their support of the main PI-institute and we are indebted to ESA for their courage in embarking on the Mars Express program, the first ESA mission to the red planet.

Ella Carlsson also wishes to thank the Swedish National Graduate School of Space Technology and the Kempe Foundation for financial support.

\section{References}

Acũna, M.H., Connerney, J.E.P., Wasilewski, P., Lin, R.P., Anderson, K.A., Carlson, C.W., McFadden, J., Curtis, D.W., Mitchell, D., Reme, H., Mazelle, C., Sauvaud, J.A., d'Uston, C., Cros, A., Medale, J.L., Bauer, S.J., Cloutier, P., Mayhew, M., Winterhalter, D., Ness, N.F., 1998. Magnetic field and plasma observations at Mars: Initial results of the Mars Global Surveyor Mission. Science 279, 5357, 1676-1680.

Barabash, S., Lundin, R., Andersson, H., Gimholt, J., Holmström, M., Norberg, O., Yamuchi, M., Asamura, K., Coates, A.,Linder, D.R., Kataria, D.O., Curtis, C.C.,Hsieh, K.C., Sandel, B.R., Fedorov., A., Grigoriev, A., Budnik, E., Grande, M., Carter, M., Reading, D.H., Koskinen, H., Kallio, E., Riihela, P., Säles, T., Kozyra, J., Krupp, N., Livi, S., Woch, J., Luhmann, J., McKenna-Lawlor, S., Orsini, S., Cerulli-Irelli, R., Maggi, M., Morbidini, A., Mura, A., Milillo, A., Roelof, E., Williams, D., Sauvaud, J.-A., Thocaven, J.-J, Moreau, T., Winningham, D., Frahm, R., Scherrer, J., Sharber, J., Wurz, P., Bolchsler, P., 2004. ASPERA-3: Analyser of Space Plasmas and Energetic Ions for Mars Express. ESA special publication SP-1240, 121-139. 
Bibring, J.-P., Langevin, Y., Gendrin, A., Gondet, B., Poulet, F., Berth, M., Soufflot, A., Arvidson, R., Mangold, N., Mustard, J., Drossart, P., and the OMEGA team, 2005. Mars Surface Diversity as Revealed by the OMEGA/Mars Express Observations. Science 307, 5715, 1576-1581.

Calvin, W. M., Hematite Spherules of Meridiani Planum: Implications for Aqueous History at the Site, American Geophysical Union, abstract \#P21A-0216, Fall Meeting 2004.

de Pater, I., Lissauer, J.J., 2001. Planetary Sciences. Cambridge Univ. Press., Cambridge, UK.

Fox, J., 2004. Response of the Martian thermosphere/ionosphere to enhanced fluxes of solar soft X rays. J. Geophys. Res. 109, A11.

Haberle, M.R., 1998. Early Mars climate models. J. Geophys. Res. 103, 28, 467-479.

Head, J.W., Heisinger, H., Ivanov, M.A., Kreslavsky, M.A., Pratt, S., Thomasn, B.J., 1999. Possible ancient oceans on Mars: evidence from Mars Orbiter Laser Altimeter data. Science 286, 2134-2137.

Hess, S.L., Henry, R.M., Tillman, J.E., 1979. The seasonal variation of atmospheric pressure on Mars as affected by the south polar cap. J. Geophys. Res. 84, 2923-2927.

Kallio, E., 1996. An empirical model of the solar wind flow around Mars. J. Geophys. Res. 101, A5, 11133-11148.

Kallio, E., Luhmann, J.G., and Barabash, S., 1997. Charge exchange near Mars: The solar wind absorption and energetic neutral atom production. J. Geophys. Res. 102, A10, 22183-22198.

Kasting, J.F., 1991. $\mathrm{CO}_{2}$ condensation and the climate of early Mars. Icarus 94, 1-13.

Kessel, R.L., Johnstone, A.D., Coates, A.J., Gowen, R.A., 1989. Space plasma measurements with ion instruments. Rev. Sci. Instr. 60, 12, 3750-3761. 
Lui, Y., Nagy, A.F., Gombosi, T.I., DeZeeuw, D.L., Powell, K. G., 2001. The solar wind interaction with Mars: results of three-dimensional three species MHD studies. Adv. Space. Res. 27, 11, 1837-1846.

Lundin, R., Zakharov, A., Pellinen, R., Borg, H., Hultqvist, B., Pissarenko, N., Dubinin, E.M., Barabash, S.W., Liede, I., and Koskinen, H., 1989. First measurements of the ionospheric plasma escape from Mars. Nature 341, 19 Oct, 609-612.

Lammer, H., Lichtenegger, H.I.M., Kolb, C., Ribas, I., Guinan, E.F., Abart, R, and Bauer, S.J., 2003. Loss of water from Mars: Implications for the oxidation of the soil. Icarus 165, 9-25.

Lundin, R., Zakharov, A., Pellinen, R., Borg, H., Hultqvist, B., Pissarenko, N., Dubinin, E.M., Barabash, S., Liede, I., Koskinen, H., 1990. Plasma composition measurements of the martian magnetosphere morphology. Geophys. Res. Letters 17, 6, 877-880.

Malin, M., Edgett, K., 2003. Evidence for Persistent Flow and Aqueous Sedimentation on Early Mars. Science 302, 1931-1934, .

Malin, M., Edgett, K., 2000. Evidence for Recent Groundwater Seepage and Surface Runoff on Mars. Science 288, 2330-2335.

Markwardt, C.B., NASA/GSFC Code 662, Greenbelt, MD 20770

http://cow.physics.wisc.edu/ craigm/idl/idl.html

Melosh, H.J., Vickery, A.M., 1989. Impact erosion of the primordial atmosphere of Mars. Nature 338, 06 April, 487-489.

Murray, J. B., Muller, J.-P., Neukum, G., Werner, S. C., van Gasselt, S., Hauber, E., Markiewicz, W. J., Head, J.W., Foing, B.H., Page, D., Mitchell, K.L., Portyankina, G., Co-Investigator Team, The HRSC., 2005. Evidence from the Mars Express High Resolution Stereo Camera for a frozen sea close to Mars' equator. Nature 434, 352-356. 
Norberg, O., Yamauchi, M., Lundin, R., Olsen, S., Borg, H., Barabash, S., Hirahara, M., Mukai, T. and Hayakawa, H., 1998. The Ion Mass Imager on the Planet-B Spacecraft. Earth, Planets and Science 50, 199-205.

Norberg, O., Lundin, R., and Barabash, S., 1993. Observations of molecular ions in the martian plasma environment. COSPAR colloquium series, 4, "Plasma environments of non-magnetic planets", edited by Gombosi, T.I., 299-304, Pergamon, England.

Penz, T., Arshukova, I.L., Terada, N., Shinagawa, H., Erkaev, N.V., Biernat, H.K., Lammer, H., 2005. A comparison of magnetohydrodynamic instabilities at the Martian ionopause. Adv. Space. Res., in press.

Ribas. I., Guinan, E.,F., Güdel, M., Audard, M., 2005. Evolution of the solar activity over time and effects on planetary Atmospheres. I. High-energy Irradiances (1-1700 ). The Astrophys. J. 622, 1, 680-694.

Wood, B.E., Müller, H-R., Zank, G.P., Linsky, J.L., 2002. Measured mass-loss rates of solar-like stars as a function of age and activity. The Astrophys. J. 574, 412-425. 
Table 1: Upward fluxes of the main ions according to Fox[2005](private communication). The third column shows the fluxes normalised to the $\mathrm{O}^{+}$flow.

\begin{tabular}{|l|c|c|}
\hline Ions & Flux in $\mathrm{cm}^{-2} \mathrm{~s}^{-1}$ & Ratio \\
\hline $\mathrm{CO}_{2}^{+}$ & $4.02 \cdot 10^{7}$ & 1.63 \\
$\mathrm{O}^{+}\left({ }^{4} \mathrm{~S}\right)$ & $2.47 \cdot 10^{7}$ & 1.0 \\
$\mathrm{O}_{2}^{+}$ & $2.34 \cdot 10^{8}$ & 9.47 \\
\hline
\end{tabular}

Table 2: The arithmetic mean ratios for $\mathrm{CO}_{2}^{+} / \mathrm{O}^{+}$and $\mathrm{O}_{2}^{+} / \mathrm{O}^{+}$compared to the ratios by Fox[2005](private communication) based on the maximum upward fluxes from the ionosphere and their confidence intervals in which $97 \%$ of the ratios can be found.

the ratios can be found.
\begin{tabular}{|l|c|c|}
\hline This study, arithmetic ratio mean, $\overline{R_{j}}$ & $\mathrm{CO}_{2}^{+} / \mathrm{O}^{+}$ & $\mathrm{O}_{2}^{+} / \mathrm{O}^{+}$ \\
Confidence interval (97\% probability) & $\mathbf{0 . 2}$ & $\mathbf{0 . 9}$ \\
Fox[2005] & $0-0.4$ & $0.4-1.4$ \\
Divergence factor (This study with respect to Fox $[2005])$ & 8.0 & 9.47 \\
\hline
\end{tabular}




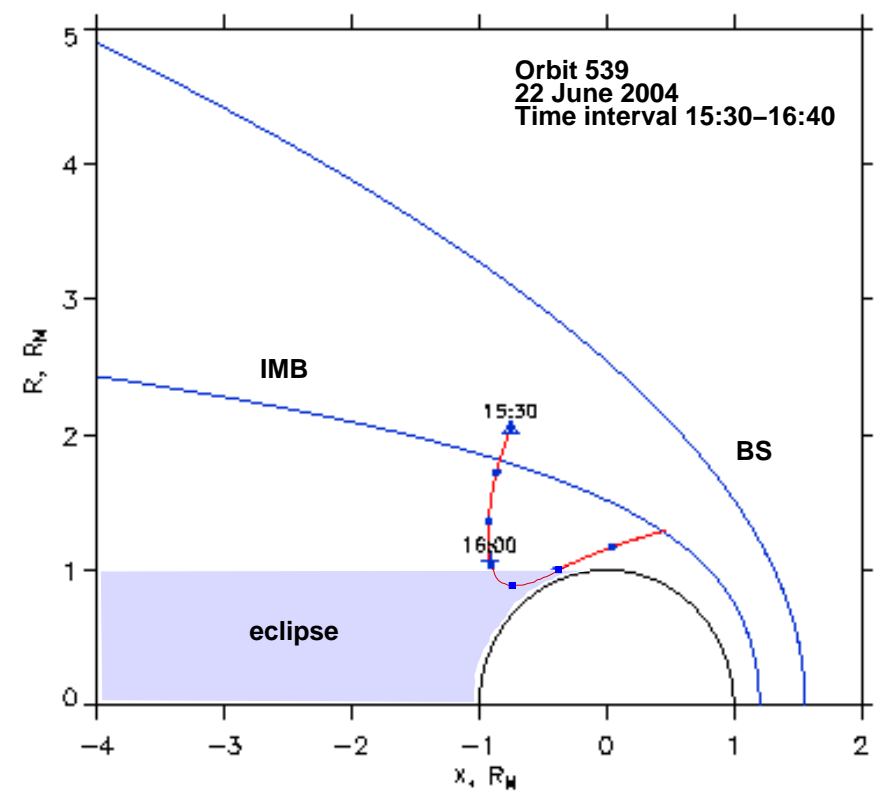

Figure 1: Orbit \#539 displayed in cylindrical coordinates normalised to the radius of Mars $\left(\mathrm{R}_{m}\right)$, showing the positions of the bow shock (BS), induced magnetosphere boundary (IMB) and the eclipse zone. The $x$-axis in this coordinate system is oriented toward the sun. The horizontal axis $\mathrm{R}=\sqrt{y^{2}+z^{2}}$, represents the distance from the satellite to the Mars-Sun line. 


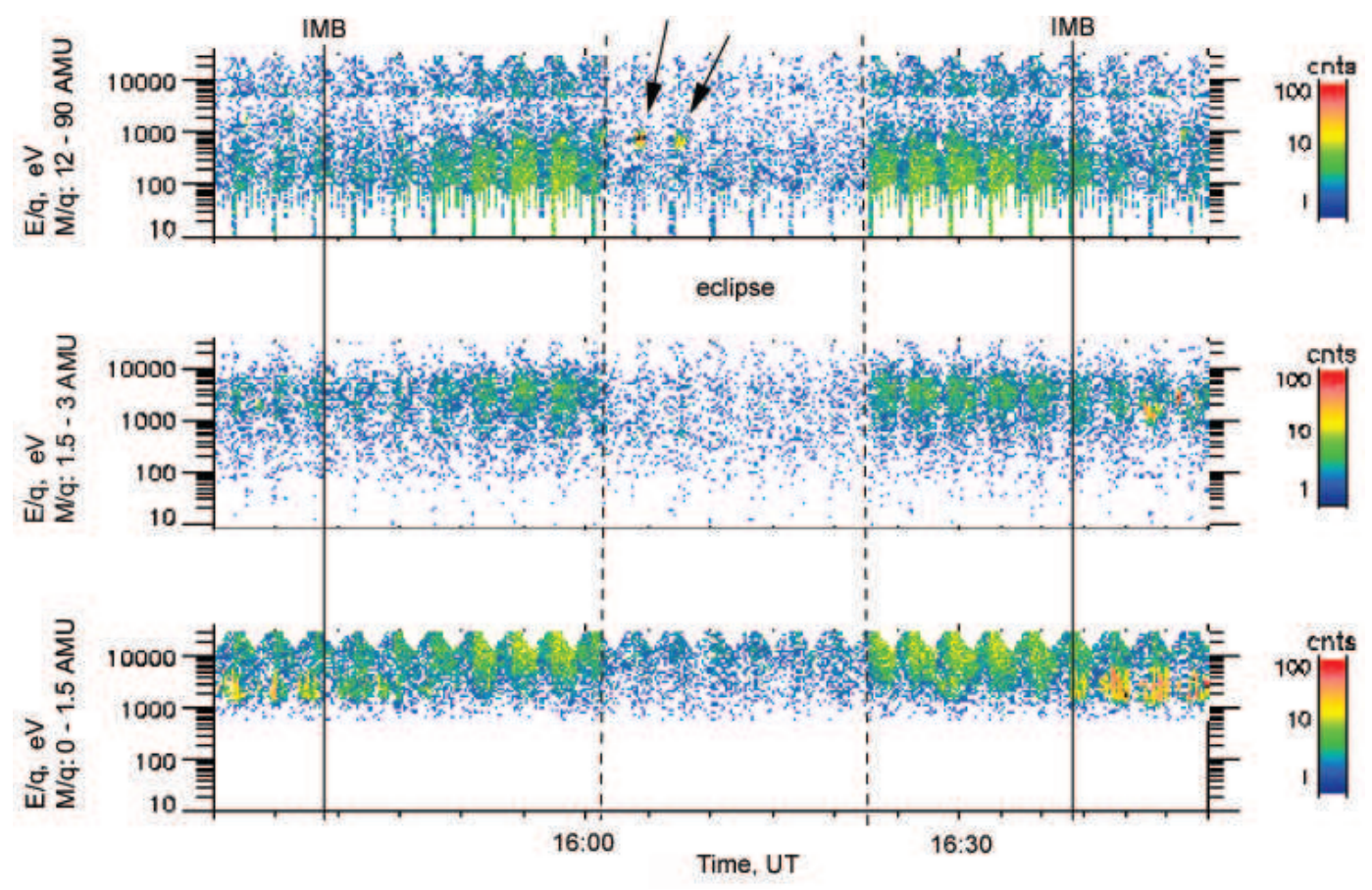

Figure 2: IMA sensor data showing energy - time spectrograms for different M/Q intervals. From bottom to top: $\mathrm{M} / \mathrm{Q}=0.0-1.5\left(\mathrm{H}^{+}\right), \mathrm{M} / \mathrm{Q}=1.5-3\left(\mathrm{He}^{2+}\right), \mathrm{M} / \mathrm{Q}=12-90$ (planetary ions). Count rates in the selected mass interval is coded by colour. In each panel the horizontal axis shows the universal time and the vertical axis shows the ion energy in $\mathrm{eV}$. The two vertical black lines indicate crossings of the induced magnetosphere boundary (IMB). The two dashed lines signify, respectively, the beginning and end of the eclipse. The identified ion-beam event is emphasised by arrows. 

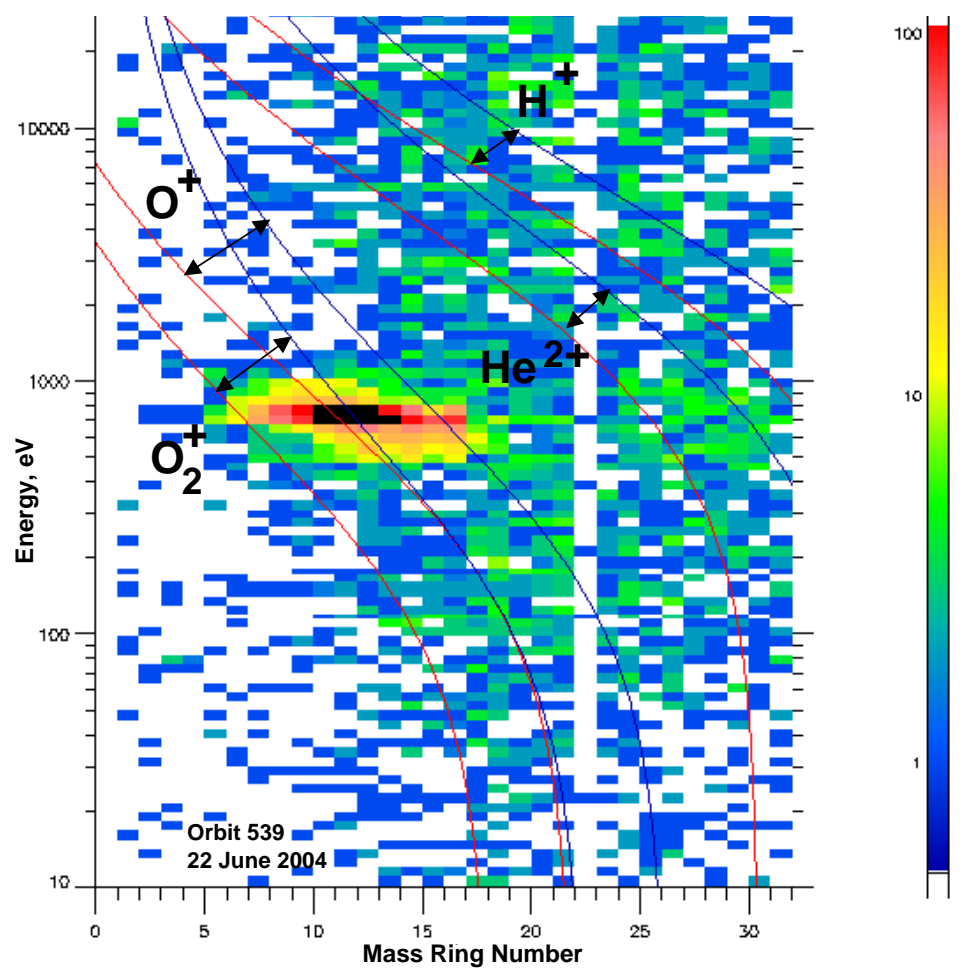

Figure 3: Energy-mass matrix of the event at orbit \#539. The vertical axis represents the energy per charge in $\mathrm{eV}$ and the horizontal axis the mass ring number. The red and blue lines show the calibrated mass band ranges for $\mathrm{O}_{2}^{+}, \mathrm{O}^{+}, \mathrm{He}^{2+}$ and $\mathrm{H}^{+}$. 

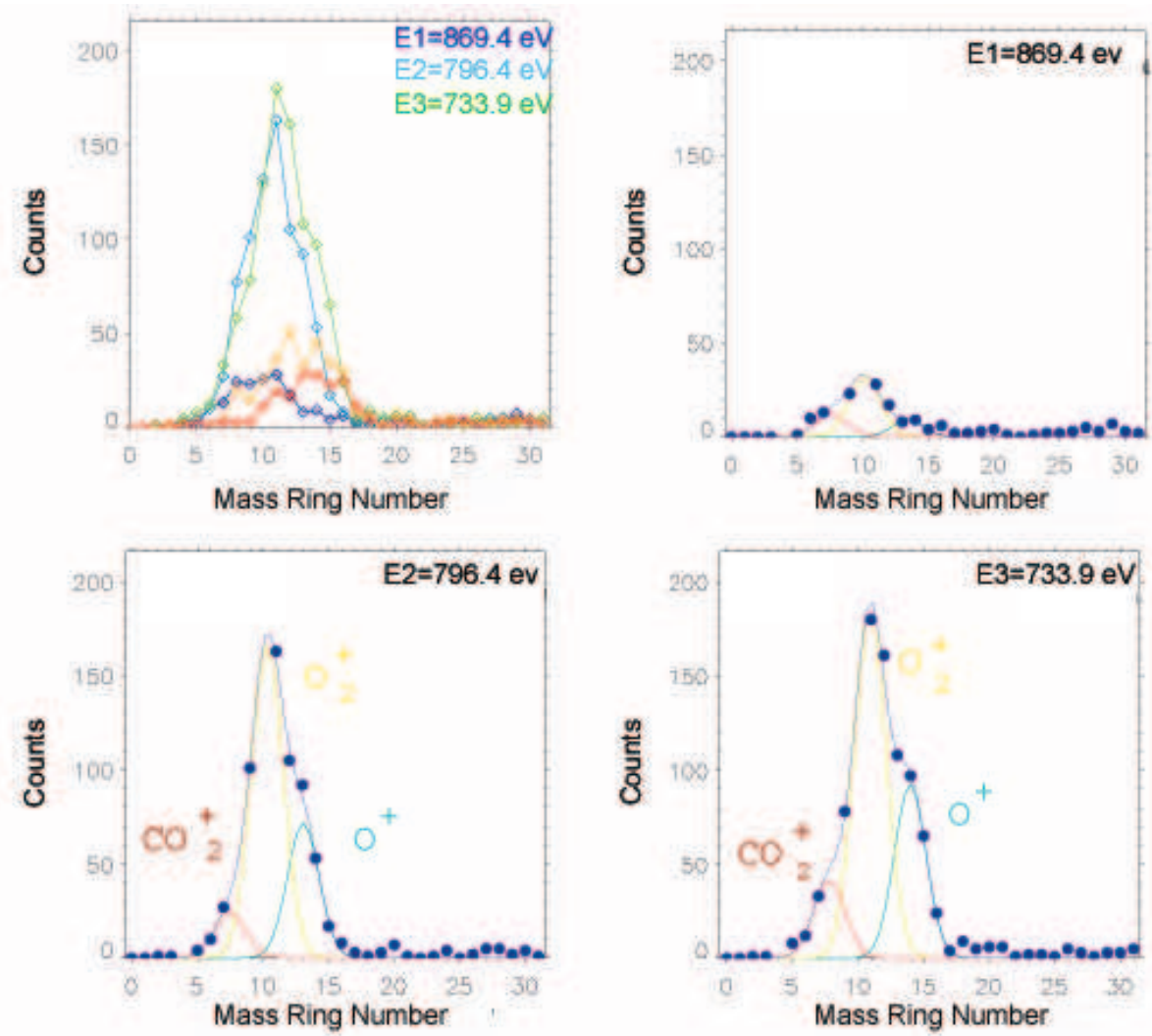

Figure 4: The top left panel shows count rate versus mass ring number for different energies for the ion-beam event that occurred during orbit \#539, 22 June 2004. The other three panels show a mass-fitting of recorded profiles of count rates for three selected energies. Best fitting is shown for the species $\mathrm{CO}_{2}^{+}(\mathrm{M} / \mathrm{q}=44)$, $\mathrm{O}_{2}^{+}(\mathrm{M} / \mathrm{q}=32)$, and $\mathrm{O}^{+}(\mathrm{M} / \mathrm{q}=16)$. 


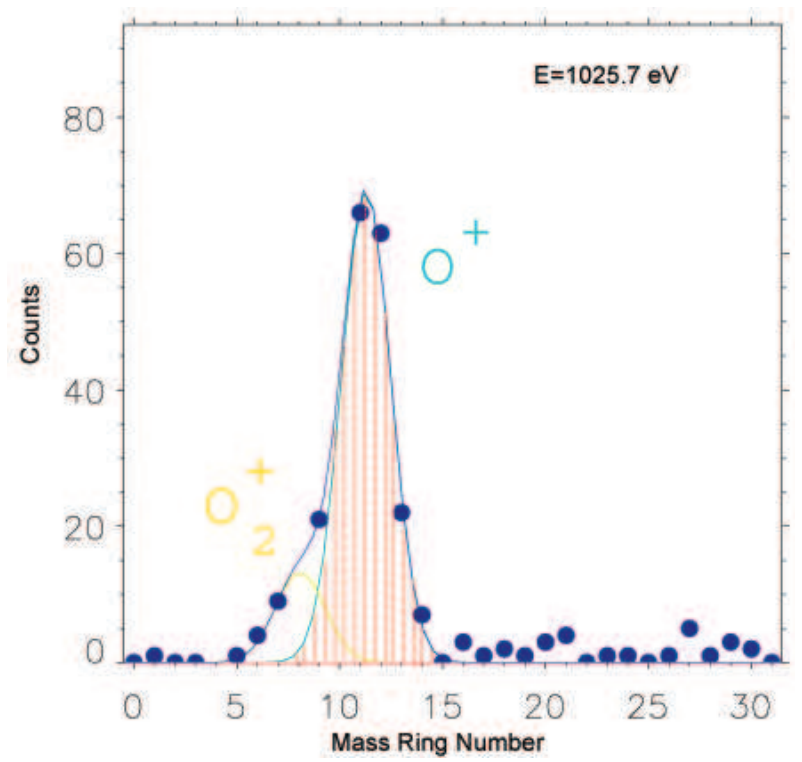

Figure 5: Mass spectrum with fitting for the ion-beam event at orbit \#495, 10 June 2004. The red lined area indicates the mass-fitted ion-species of $\mathrm{O}^{+}$. The spectrum was integrated over the time interval of 18 minutes.

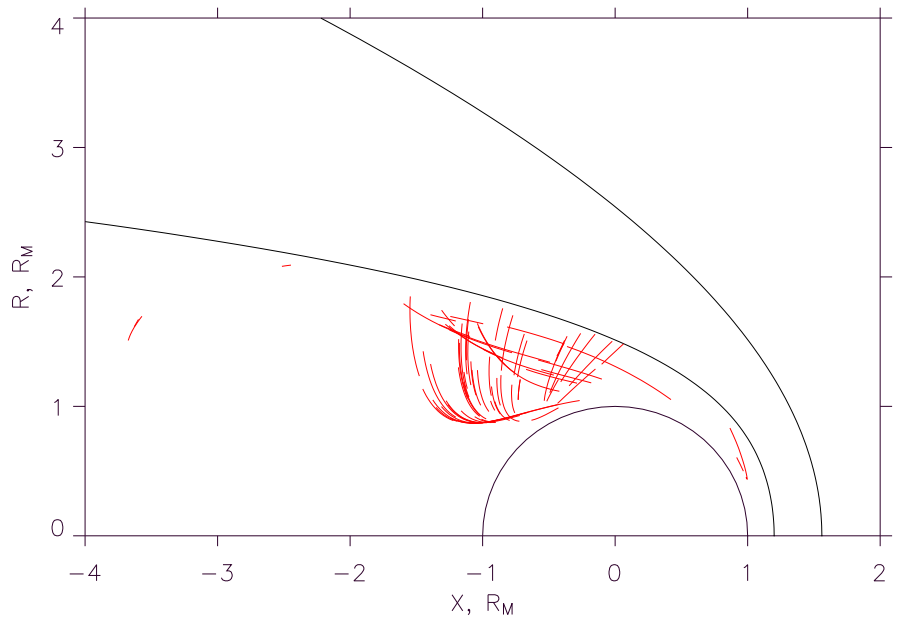

Figure 6: Pieces of the orbits corresponding to the time intervals of all ion-beam events used in this study. 

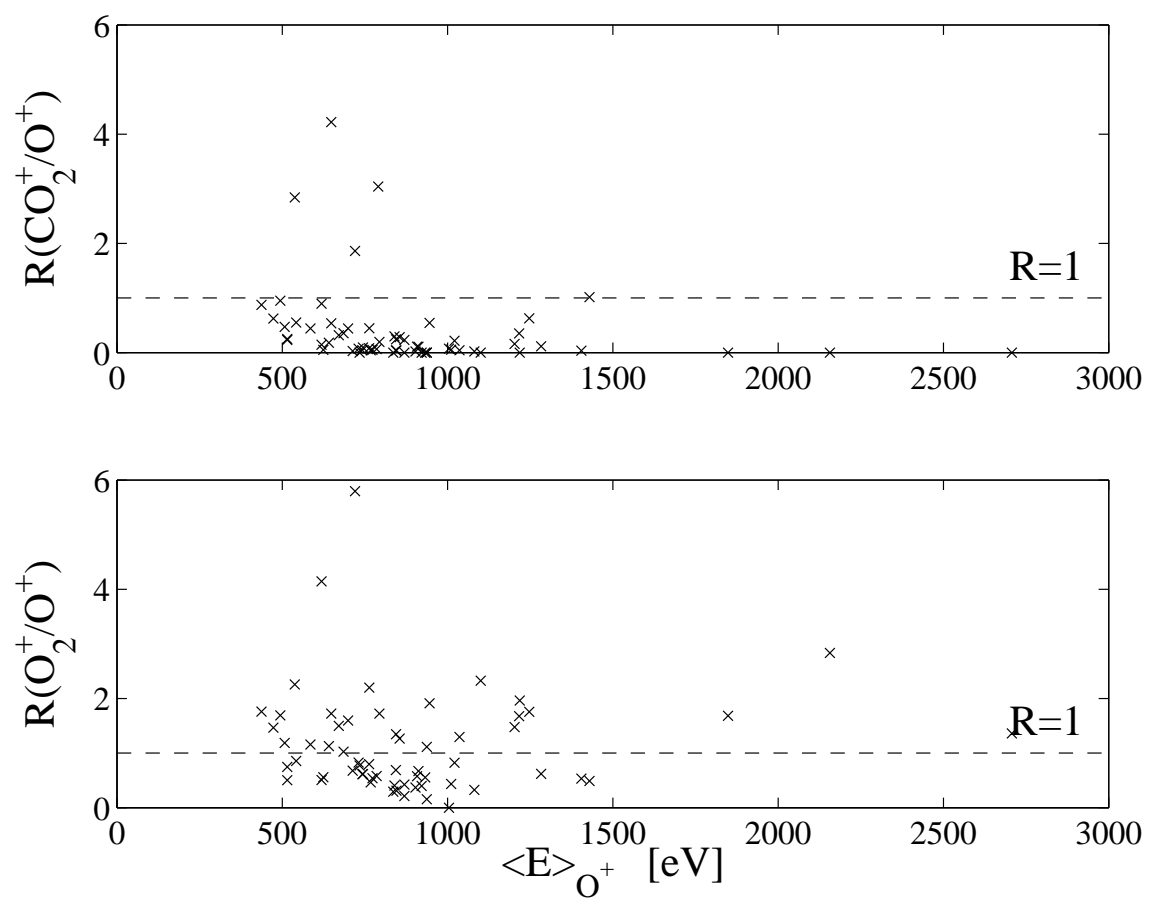

Figure 7: Ratios of the $\mathrm{CO}_{2}^{+} / \mathrm{O}^{+}$and $\mathrm{O}_{2}^{+} / \mathrm{O}^{+}$fluxes related to energy. The vertical axis in each panel shows the $\mathrm{CO}_{2}^{+} / \mathrm{O}^{+}$and $\mathrm{O}_{2}^{+} / \mathrm{O}^{+}$ratios for each event, while the horizontal axis shows the mean energy in eV for each event. 


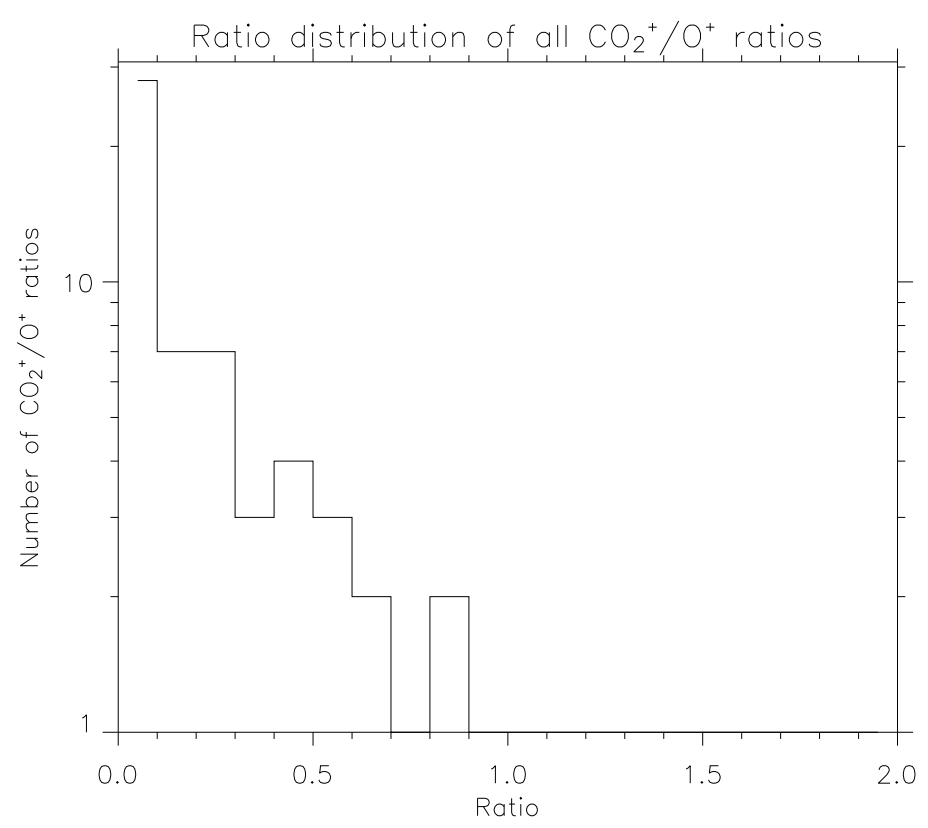

Figure 8: Ratio distributions of $\mathrm{CO}_{2}^{+} / \mathrm{O}^{+}$. The vertical axis represents the number of ratios within each bin in a logarithmic scale and the horizontal-axis represent the ratio values within bins of size 0.1.

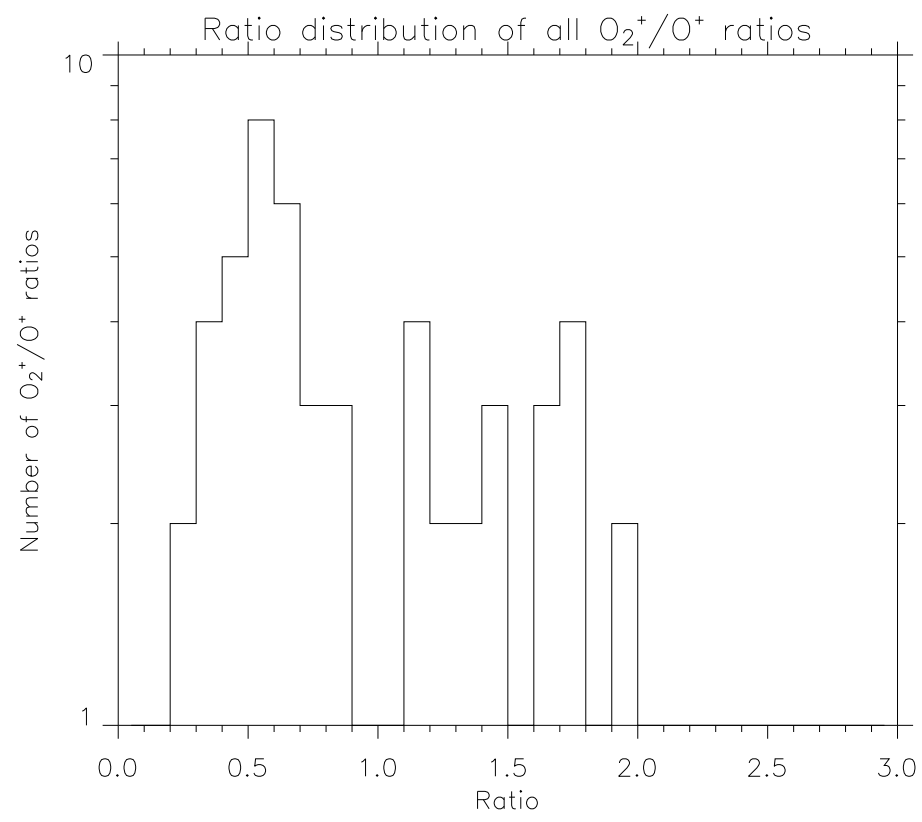

Figure 9: Ratio distributions of $\mathrm{O}_{2}^{+} / \mathrm{O}^{+}$. The vertical axis represents the number of ratios within each bin in a logarithmic scale and the horizontal-axis represent the ratio values within bins of size 0.1. 